\title{
Facts on fluoridation
}

If we had vehemently opposed water fluoridation on the grounds it was taking away our livelihood then there is a strong chance everyone would have fought "tooth and nail' to ensure it happened.

Mike Grace m.grace.bdj@bda-dentistry.org.uk

\begin{abstract}
n his book The Reluctant Messiah Richard Bach (the author of Jonathan Livingstone Seagull) writes this simple sentence, 'Beliefs can be ferocious traps.' Indeed they can, as we will doubtless see in the reaction to the recently published systematic review on water fluoridation.
\end{abstract}

The research was carried out by the NHS Centre for Reviews and Dissemination at York University. A search of 25 electronic databases and the world-wideweb was undertaken, providing a total of 214 studies that met the criteria for inclusion, made up of 45 'before and after' studies, 102 cross-sectional studies, 47 ecological studies, 13 cohort (prospective and retrospective) studies and 7 case-control studies. The results showed that water fluoridation reduces caries in children by on average 2.25 teeth per child, and increases by $15 \%$ the proportion of children completely free from tooth decay.

The researchers also found evidence that water fluoridation reduces inequalities in dental health by narrowing the dental health gap between young children living in poverty and their more affluent peers. They found no evidence to support claims that water fluoridation caused any harm. On fluorosis, they estimate that fluoridation would slightly increase the prevalence of dental fluorosis of 'aesthetic concern'. Finally they found no difference between naturally and artificially fluoridated water.

So now we have the facts. Reaction from all sides of the debate is predictable, with the pro-fluoride faction triumphantly reporting the lack of evidence for harm and the confirmation that water fluoridation actually works. The anti-fluoridationists claim the report is biased and does not provide absolute proof because of the studies that were supposedly 'excluded'. The 'Greens' are simply opposed to fluoridation, though apparently not on the grounds it could damage the environment (no change there, then). In short - we are no further on unless someone with authority (the government) does something about it.

But will they? As long as people continue to let their beliefs control their behaviour (which is how we actually work) it seems unlikely. After all, beliefs really are ferocious traps (as Richard Bach so succinctly put it), especially in the areas of health behaviour. How else can we explain the continued acceptance of alcohol by virtually everyone on the planet, the rise in the incidence of smoking in the UK again and the almost anarchic reactions to genetically-modified food? Who really cares about the evidence? If I feel it is right/wrong (delete whichever does not apply) in 'my bones' then no amount of scientific evidence or hard facts will persuade me otherwise. After all, how do I know it's not all a conspiracy anyway?

It is perhaps a little frustrating to see the same old arguments rolled out again on both sides. For water fluoridation the answer is simple - the government must legislate (as it promised originally and is now appearing to backtrack on). Nothing else will work. We know that $70 \%$ of the population surveyed independently want water fluoridation. We know that current legislation gives Health Authorities responsibility to recommend water fluoridation if required for the people in their area. We also know water companies can and do currently prevent water fluoridation, and that only a change in the law will rectify the current impasse. After all, the water industry itself would welcome it.

Perhaps the real answer is that dentistry originally chose the wrong strategy (as was once suggested to me half in jest). If we had vehemently opposed water fluoridation on the grounds that it was taking away our livelihood then there is a strong chance everyone would have fought 'tooth and nail' to ensure it happened. Such is the way people work. Unfortunately for the population dentists are not devious enough. 